\title{
ORIGENES, DESARROLLO HISTORICO Y ESTADO ACTUAL DE LOS HERBARIOS EN EL MUNDO
}

\author{
Fernando Medellín-Leal ${ }^{*}$, *
}

\section{1.--LOS ORIGENES}

¿Cuándo comenzó el hombre a conservar plantas o partes de plantas? Dado que para el hombre los vegetales son base de su alimentación y fuente de un gran número de dichas (comidas, bebidas, drogas, etc.) y desdichas (comidas, bebidas, drogas, etc.) es muy posible que su afición por conservar plantas haya comenzado desde su aparición como tal, es decir como hombre.

Podemos ver en nuestra imaginación al primitivo recolector reuniendo y guardando en su guarida, un tanto a la manera de algunos animales, provisiones para la temporada adversa. Muy pronto debe de haber aprendido que algunas plantas o partes de ellas se conservan mejor que otras. Más tarde, con el advenimiento de la agricultura, la conservación de los granos no sólo le aseguraba una buena proporción de su alimento, sino que, seleccionando cada vez un poco más podía hacer efectiva la repetición de un ciclo que poco a poco le iba asegurando su incipiente señorío sobre la naturaleza; podía así tener potencialmente el futuro a su favor no sólo por un ciclo anual sino, en algunos casos sería capaz de asegurarse por muchos años más, suponiendo que el clima no lo traicionara.

Pronto, históricamente hablando, debe también haber aprendido en base a experiencias concientes o inconcientes cuáles plantas, aparte de las alimenticias, le ayudaban a sentirse mejor, cuáles lo curaban de sus enfermedades reales o imaginarias, cuáles le producían placer, cuáles le proporcionaban seguridad en el ataque o en la defensa y, por último, cuáles podían causarle la muerte.

Pero esto, bien entendido, no es sino un pasaje remoto de la humanidad, cuyos fines eminentemente prácticos parecen, a primera vista, no tener gran relación con el tema que hoy tratamos. Podemos por lo tanto, dar marcha

* Instituto de Investigación de Zonas Desérticas, Universidad Autónoma de San Luis Potosí. San Luis Potosí, S.L.P.

** El autor agradece al Hno. José Isaak M.Sp.S., Licenciado en Historia, la revisión crítica del manuscrito así como sus valiosas sugerencias. 
atrás y volver a nuestra pregunta inicial, ampliándola un poco para, a la vez, restringirla.

¿Cuándo comenzó el hombre a conservar plantas o partes de plantas con fines científicos? La respuesta es bastante similar a la anterior. Probablemente desde la aparición de la ciencia como tal, entendida ésta como función especulativa racional y desligada hasta lo posible de interpretaciones mágico-religiosas, variables según el grado de cultura de los pueblos y decimos "hasta lo posible", porque la historia de la ciencia en general y de la botánica en particular nos enseña que esta unión ciencia-magia-religión o viceversa, para seguir una secuencia más histórica, religión-magia-ciencia persiste aún en determinados rincones de nuestra conciencia personal y/o colectiva.

Las primeras colecciones de seres vivos que iniciaron los científicos primitivos estaban constituidas de manera fundamental precisamente por eso, por ser seres vivos en el sentido corriente de la palabra y así, vemos que las primeras colecciones de plantas con un fin premeditado de servir a reflexiones filosóficas y a la enseñanza de futuras generaciones, se hicieron fundamentalmente de plantas vivas. Los jardines botánicos (Hortus) preceden por muchos siglos a las colecciones de plantas secas, que por la convergencia de objetivos con sus antecesores fueron en sus inicios llamados Hortus siccus (jardines secos) los que actualmente conocemos como Herbarios.

Los jardines botánicos, cuyos orígenes se pierden en la obscuridad de los tiempos, son por lo tanto los antepasados más próximos y ascendientes en línea directa de nuestros modernos herbarios. Esto no debe sorprendernos, ya que parece ser un resultado lógico del avance de la cultura, pues se repite sistemáticamente en pueblos muy separados entre sí en el tiempo y en el espacio.

Ocurrió en la Mesopotamia, se repitió en Egipto, apareció de nuevo en Europa y a muchos siglos de distancia el proceso se reproduciría en América entre los aztecas y los incas que seguramente hubieran llegado por sí solos al establecimiento de herbarios a no ser por el descubrimiento de América y su impacto sobre las culturas autóctonas.

Así pues, los jardines botánicos, como padres, y los herbarios, como hijos, tienen una estrecha relación entre sí. Muchos de los más famosos o más antiguos herbarios que funcionan en la actualidad tuvieron precisamente un jardín botánico como cuna. Si uno se preguntara ¿pero por qué razón los jardines botánicos preceden a los herbarios y no al revés, siendo que estos últimos son más fáciles de manejar y su conservación resulta mucho más económica en relación al volumen de material conservado?

Las respuestas llegan en tropel: Primeramente, y a nivel de la ciencia primi- 
genia, referida por lo que respecta a la botánica a un espacio geográfico relativamente pequeño, los jardines botánicos requerían para representar la flora de determinada zona no mucho espacio y el cuidado de, siempre relativamente hablando, pocos ejemplares.

En segundo lugar, su manejo no era realmente costoso pues existía la esclavitud y además tal actividad tenía escasa competencia dado por una parte que al pueblo, como en todos los tiempos, le importaba muy poco lo que hacían los botánicos y por otra, que estos trabajos eran patrocinados generalmente por el gobierno que buscaba, como en el caso del hombre primitivo, una aplicación práctica, productiva e inmediata. Situaciones ambas que desgraciadamente per. sisten hasta nuestros días.

En tercer lugar hay que considerar el punto de vista de los primeros naturalistas; las cosas vivas hay que conocerlas, verlas y estudiarlas precisamente vivas. Es curioso, pero el hombre "respetable" al identificarse él mismo como ser dotado de vida no se animó por muchísimo tiempo, salvo en determinadas condiciones, quizás por respeto (¿o por miedo?) a trabajar y estudiar sistemáticamente sobre cadáveres, así fueran éstos de plantas, para encontrar en el ser muerto claves indicadoras de las funciones del ser vivo.

En cuarto lugar, no es de esperar que en un tiempo en el que casi no se conocían métodos de preservación adecuada, el hombre se preocupara mucho por conservar objetos que de antemano sabía se le iban a descomponer, aunque en el caso de los vegetales esta dificultad fue salvada quizás desde antes de la aparición de la ciencia y de ello da testimonio fidedigno la universal y desde el principio de los tiempos, floreciente actividad de los yerberos o herbolarios, que en curiosa mezcla mágico-empírico-científica han realizado un activo comercio basado principalmente en plantas o partes de plantas secas, conservadas en tal forma y con tal cuidado que resisten bien el paso de los años.

En quinto y último lugar, pero quizás como principal razón, los avances de la cultura, de la ciencia botánica y por ende de los herbarios han ido mejorando y/o modificando sus técnicas. Por ejemplo una planta del jardín botánico de Teofrasto, para no mencionar sino al más clásico de los botánicos, quizás se trataría de conservar seca únicamente para refrescar, de una manera vaga, la memoria del maestro o como una referencia también vaga para sus alumnos o para la hora de redactar sus escritos, pero realmente no importaba en absoluto que dicha planta seca fuera o no fuera un "buen ejemplar de herbario" según el criterio moderno, pues además de que los herbarios no existían, difícilmente podía ocurrírseles que el ejemplar mostrara elementos absolutamente necesarios para la taxonomía si ésta tampoco existía aún y si por ejemplo, toda- 
vía no se sospechaban y mucho menos se conocían las verdaderas funciones de los diferentes órganos de las plantas (raíz, tallo, hojas, flores, etc.). Es cierto que los trabajos clásicos fueron los cimientos para el ulterior desarrollo de la botánica, pero los botánicos de aquella época no tenían la menor idea de estarlos construyendo. Incidentalmente y apartándonos un poco del período clásico podemos indicar que los botánicos y con ellos los herbarios, tienden a seguir la moda de los tiempos (característica que compartimos con toda técnica y toda ciencia). En la actualidad por ejemplo no es raro encontrar un herbario constituido exclusivamente por diatomeas o por granos de polen; situación que no podía darse antes de la invención del microscopio, o para dar otro ejemplo, un herbario fotográfico (fotografías comunes, microfichas, micropelículas, transparencias, etc.) no podía existir antes de la aparición de la cámara fotográfica.

\section{2.-EDAD MEDIA}

Pero sigamos con nuestra historia: El último de los botánicos del período clásico fue seguramente Dioscórides, quien describió y muy posiblemente dibujó un gran número de plantas, principalmente aquellas que podían tener un uso inmediato; y a él siguió la larga y nebulosa Edad Media a la que Singer llama "Edad del Oscurantismo", 1 aunque en lo personal no estamos totalmente de acuerdo con esta definición; durante la cual los escolásticos basados en Dioscórides elaboraron aquellos famosos "herbarios pictóricos" (Hortus pictus), en los que al correr de los siglos y a fuerza de copiarse unos a otros hasta el infinito, sin acercarse a mirar en la naturaleza las plantas que se suponía estaban representando, acabaron en el mejor de los casos transformando las fresas en zarzamoras y en el peor, plantas que seguramente eran de uso corriente, en objetos totalmente desconocidos. ${ }^{2}$

\section{3.-DEL RENACIMIENTO A LA ACTUALIDAD}

Pero todo termina y la Edad Media terminó con la explosión cultural del Renacimiento y aquí como en otros muchos casos fueron, para la botánica, las artes las que marcaron la pauta y los caminos a seguir; especialmente la pintura y así podemos ver que ya desde la primera mitad del siglo xv cuando nuestra

1 Singer, Charles. Historia de la Biología. Máximo Valentinuzzi trad. Buenos Aires, Es. pasa-Calpe, 1947. p. 100.

2 Singer (op. cit., pp. 101-106). 
ciencia todavía no entraba de lleno en su proceso de renovación, algunos pintores (Van Eyck, Botticelli, etc.) apartándose de los escolásticos logran obras maestras de observación de la naturaleza; Van Eyck por ejemplo en su famoso políptico conocido como "El cordero místico" (1432) nos da en varias escenas más de 40 plantas absolutamente identificables no sólo hasta especie sino que algunas de ellas inclusive hasta variedad. ${ }^{3}$ Por su parte, Botticelli en su conocida alegoría de "La primavera" (1478) representa una gran cantidad de vegetales, de los cuales 30 son reconocibles, como en el caso de Van Eyck hasta especie. ${ }^{4}$ Pero no olvidemos que también la escultura contribuyó en buena parte a esta fase de renovación y de regreso a la naturaleza, pues inclusive desde la Edad Media los artesanos de las hermosas construcciones góticas, sin conocer o a pesar de conocer los trabajos confusos de los escolásticos, habían representado en los edificios que ornamentaron bellas reproducciones fidedignas de las plantas que los rodeaban, principalmente de aquellas que tenían un significado místico, tales como parras, uvas y trigo. ${ }^{5}$

Por otro lado, Europa había recibido de los árabes, vía España, el gran patrimonio que significaba la posibilidad de producir papel y de España esa producción de papel en relativamente gran escala pasó a Italia. ${ }^{6}$ Del Norte llegó el invento de Gutenberg y con el mismo y con el papel que rápidamente difundía las ideas y que era un excelente vehículo para secar las plantas, existieron por primera vez las facilidades mínimas indispensables para que los herbarios nacieran y comenzaran a trabajar.

El primer jardín botánico del Renacimiento se fundó en Pisa en 1543 a él le siguieron el de Padua y el de Florencia ambos en $1545 .^{7 *}$ Se atribuye a un oscuro médico bolonés llamado Luca Ghini la creación del primer herbario ${ }^{8}$ precisamente en Pisa pero de este herbario no quedan rastros. Luca Ghini no

${ }^{3}$ Dierick, Alfons. Van Eyck; L'Agneau Mystique. Gand, Daphne, 1958. pp. 5, 9, 18 , $23,27,29,43$.

${ }_{4}^{4}$ Ricci, Corrado comp. Album de las galerías de pinturas de los muscos de Florencia Domingo Miral y López trad. Barcelona, Labor, s.f. lám. 60.

5 Pierre, André Louis. Cathédrales de France, Arts-Techniques-Societé. Parıs, Les Pro ductions de Paris et Pierre Amiot eds, 1962. 432 y.

${ }_{6}$ Paper; History. In The Encyclopedia Americana; The international reference work. New York, American Corporation, c 1961. v. 21, pp. 258-259.

7 Lanjouw, J. and F. A. Stafleu. Index Herbariorum; Part I, The herbaria of the world. 4th ed. Utrecht, International Bureau for Plant Taxonomy and Nomenclature of the International Association for Plant Taxonomy, 1959. 249 p. (Regnum Vegetabile v. 15).

8 Rickett, H. W. Plant Systematics. In William. D. Halsey ed. Collier s Encyclopedia. s.1., Crowell-Collier, 1964. v. 19, pp. 109-126.

* Frecuentemente se cita al Herbario de Padua como el más antiguo del mundo, sin embargo, según declaración del mismo Herbario (7), su fundación data de la fecha anotada. 
escribió nada sobre botánica y la única obra que de él se conserva es un pequeño tratado de medicina al cual no se le concede mucha importancia, sin embargo, era un estudioso admirable de las plantas y un extraordinario maestro. ${ }^{9} \mathrm{Su}$ mejor discípulo en este campo fue sin duda Ulises Aldrovandi, ${ }^{10}$ de quien se conoce más su obra zoológica; a pesar de que sus trabajos botánicos no son nada despreciables y de que sus colecciones botánicas, es decir, su herbario, lo donó al morir a la ciudad de Bolonia, ${ }^{11}$ en cuya Universidad y basándose en dicha colección se fundó por vez primera un herbario, ya como tal, en el año de 1567, mismo que se conserva hasta la actualidad. ${ }^{12}$

En esta forma Pisa y Bolonia se disputan la cuna de los herbarios. Nosotros, en particular, consideramos que ambas merecen dicho reconocimiento una por ser el lugar en donde las técnicas de herborización se inventaron y la otra por ser la que primero inició la conservación a largo plazo y por ser la que guarda los ejemplares de herbario más antiguos que se conocen; precisamente los de Ulises Aldrovandi.

Después de establecido el primer herbario continuó durante cuatro siglos una lenta pero segura creación de nuevas instituciones, debido tal vez a la influencia que la imprenta había ejercido como difusora de ideas. A la fundación de los herbarios siguió un interés creciente en los estudios botánicos.

Pero la botánica como todas las ciencias se inició con pasos vacilantes y varias veces tuvo que modificar sus técnicas de trabajo para que fueran de acuerdo con la época y sobre todo tendiendo hacia un sentido más preciso del conocimiento, en la que cada investigador fue contribuyendo en su oportunidad con pocas o muchas ideas que hacían corregir el rumbo.

Hemos de llegar a la época de los genios naturalistas: Cesalpino y los Bauhin en el siglo xvi, John Ray en el siglo xvir y sobre todo Linneo y Jussieu en el siglo xviII, verdaderos revolucionarios de la ciencia de su tiempo, para que se iniciara un rápido ascenso en el número de herbarios establecidos y es así como vemos que a partir de 1750 principia una curva creciente de fundación de este tipo de instituciones, que se continúa en forma casi vertical hasta nuestros días.

9 Ghini, Lucas. In Enciclopedia Universal Ilustrada Europeo-Americana. Madrid, Espasa-Calpe, c1924. v. 25 , p. 1535.

10 Ghini, Lucas. In Diccionario Enciclopédico Hispano-Americano de Literatura, Ciencias, Artes, etc. Barcelona, Montaner y Simon eds., s.f. v. 10, p. 372.

11 Nordenskiöld, Erik. Evolución histórica de las ciencias biológicas. Justo Garate trad. Buenos Aires, Espasa-Calpe, 1949. p. 118.

12 Lanjouw, J. and F. A. Staflcu. (op. cit., P. 30 ). 


\section{4.--LAS COLECCIONES}

Llegados a este punto podemos preguntar ¿Qué se ha guardado y qué se guarda en los herbarios del mundo? La respuesta sería obvia, puesto que la palabra "Herbario" nos sugiere ya que se trata de una colección de hierbas, $o$, generalizando, una colección de vegetales lo más completa posible; sean hierbas, arbustos, árboles o criptógamas y, de ser factible, todas las partes de dichas plantas.

Pero desde el principio pudo comprobarse que esto quedaba fuera de los alcances prácticos de las instituciones y que algunos elementos de la planta son únicamente necesarios en caso excepcionales; por ejemplo, las raíces de la mayor parte de las fanerógamas son en general difíciles de colectar, difíciles de conservar, ocupan mucho espacio y son prácticamente inútiles en la taxonomía; y ya que tocamos la palabra "taxonomía" hemos de recordar al hacer la historia de los herbarios, que si la taxonomía ha logrado nacer, desarrollarse y evolucionar, ha sido debido precisamente a la existencia de herbarios en cuyas colecciones ha encontrado la fuente de toda su inspiración, por la posibilidad de establecer semejanzas, diferencias y comparaciones, siendo testimonio fiel para sus elucubraciones. El círculo se cierra al descubrir que a su vez y desde un principio los herbarios se han apoyado en la taxonomía, la cual ha ido indicando según la época el tipo de documento que desea le sea proporcionado por el herbario, indicando al coleccionista las series de plantas o partes de las mismas que debe buscar para que sus ejemplares sean útiles, así como los datos suplementarios que espera encontrar anotados en la etiqueta que acompaña a cada muestra.

Por lo que respecta a la etiqueta; ésta, posiblemente, es el mejor ejemplo de la forma en que ha ido evolucionando el manejo de un herbario. Al principio se limitaba casi exclusivamente al nombre de la planta que el botánico había tenido a bien adjudicarle; dicho nombre, en muchos casos, no tiene nada que ver con la estructura actual de los nombres específicos puesto que la taxonomía no había alcanzado el nivel tan sutil que ahora tiene y además no había reglas de nomenclatura. Más tarde se asentó la localidad de la que provenía el ejemplar, aunque sólo fuera de una manera vaga, pues las comunicaciones eran difíciles y para un herbario que no sabía muy bien lo que buscaba era suficiente en algunos casos con mencionar el continente. En el herbario de París hemos tenido oportunidad de revisar ejemplares del siglo xviII cuya loca. lidad marcada era solamente "América"; acaso, tratando de precisar, "América 
Boreal" y en un alarde de exactitud (para esos tiempos) una etiqueta mostraba como localidad "Nova-Hispania".

Estudiando los textos de Cavanilles de principios del siglo $\mathrm{XIX}^{13}{ }^{13}$ podemos constatar que más tarde se agregó como referencia el nombre del colector y en forma aproximada la fecha. He aquí un ejemplo: "Verbena urticifolia Lin... florece por Julio: se cría en la Nueva España, donde la encontró D. Luis Nee y se cultiva en el Real Jardín Botánico". ${ }^{14}$

Poco a poco las etiquetas se han ido complicando y a las sencillas de la Antigüedad se fueron agregando: fecha exacta de colecta; localidad exacta, en donde se ha llegado a anexar un mapa de la zona, marcado con coordenadas geográficas, a veces también un corte vertical si la colecta ha sido hecha en un lugar con desniveles topográficos, e inclusive una descripción lo más detallada posible de la fisiografía; tipo de suelo; exposición; abundancia y dominancia; habitat; altitud sobre el nivel del mar; nombre completo del colector; número de colecta; nombre de la persona que ha identificado la muestra; fecha en que lo hizo; nombre de la persona que montó el ejemplar; fecha en que esto sucedió y observaciones varias que pueden ser el color de las flores, el vigor aparente de la planta, tamaño de la planta completa, el nombre vulgar y los usos que de ella hacen los nativos.

Pero volviendo a las colecciones. Los herbarios han sido desde siempre instituciones generadoras de ideas, es decir, "vivas" sin poder por lo tanto dejar de tener relaciones históricas, relaciones geográficas, relaciones culturales y relaciones sociales. El mundo que los rodea es siempre cambiante y los herbarios han sobrevivido gracias a su posibilidad de evolución por su capacidad de revisión y actualización de objetivos, modificación de sus estructuras y adaptación a las exigencias cada vez más grandes de todos los sectores sociales.

Las colecciones han ido variando de lo general a lo particular y siempre han mostrado un gran paralelismo con el desarrollo cultural. Así vemos que, iniciados en la Edad Media como libros iconográficos, fueron después colecciones de plantas secas pero no prensadas, plantas secas y prensadas o "esqueletos" como los llama Cavanilles ${ }^{15}$ y simultáneamente a estas últimas, colecciones en líquidos preservadores. No hace mucho tiempo se acostumbraban también las colecciones de elementos artificiales, tales como flores de papel y tela, de cera, de vidrio y aun de porcelana, a veces de tamaño natural; o puestos ya en el camino de

13 Cavanilles, Antonio Josef. Descripción que D. Antonio Josef Cavanilles demostró en las lecciones públicas del año 1801, precedido de los principios elementales de la botánica. Madrid, Tmprenta Real, 1802. CXXXVI+ $285 \mathrm{p}$.

1 Cavanilles (op. cit., p. 67).

15 Cavanilles (op. cit.). 
imitar para facilitar el estudio, se exageraba uno o más caracteres. Conocimos algunos de estos ejemplares en el antiguo "Museo del Chopo" y para no ir muy lejos, en el Herbario Nacional cuando el Instituto de Biología de la UNAM estaba hospedado en la Casa del Lago de Chapultepec.

Estas colecciones de elementos artificiales han ido, por lo general, quedando en el olvido pues aparte de ocupar demasiado espacio representaban en el mejor de los casos, como los "Hortus pictus" medievales, una mala caricatura de la naturaleza.

En la actualidad los herbarios del mundo van desde los muy generales, que intentan (a largo plazo, se entiende) llegar a reunir toda la flora del mundo, hasta los Herbarios que podríamos llamar "regionales" pues trabajan sobre la flora de un país, de un estado o departamento, de un municipio, condado o comuna; a veces más modestamente, de un valle, de una pequeña sierra o regiones aún más pequeñas. Hay también en la actualidad una cierta tendencia hacia la especialización en el objeto de estudio, como puede verse en la siguiente relación obtenida de un análisis cuidadoso de los datos proporcionados por Lanjouw y Stafleu en su "Index Herbariorum". ${ }^{26}$ Aparte de los herbarios muy generales o regionales, existen en el mundo herbarios que se dedican en forma más o menos exclusivas a coleccionar:

* Hongos en general. 14 herbarios

* Plantas hortícolas en general. 4 herbarios

* Algas y otras criptógamas similares. 4 herbarios

* Algas marinas exclusivamente. 4 herbarios

* Briofitas exclusivamente. 2 herbarios

* Hongos micorrízicos exclusivamente. 2 herbarios

* Hongos parásitos en general. 2 herbarios

* Plantas parásitas de otras plantas en general. 2 herbarios

* Arroz exclusivamente. 1 herbario (319 ejemplares)

* Briofitas, Pteridofitas y Gimnospermas. 1 herbario

* Comunidades vegetales. 1 herbario

* Diatomeas. 1 herbario

* Esporas y polen exclusivamente. 1 herbario

* Fotografías de ejemplares "tipo". 1 herbario (45,000 ejemplares)

* Forrajes en general. 1 herbario

* Gramíneas en general. 1 herbario

* Hongos de importancia militar. 1 herbario

16 Lanjouw and Stafleu (op. cit.). 
* Liquenes, briofitas y hongos. 1 herbario.

* Líquenes exclusivamente. 1 herbario

* Madera, frutos y semillas. 1 herbario

* Madera y corteza exclusivamente. 1 herbario

* Orquídeas exclusivamente. 1 herbario

* Plantas desérticas exclusivamente. 1 herbario

* Plantas comestibles exclusivamente. 1 herbario

* Plantas fósiles exclusivamente. 1 herbario

* Plantas indicadoras en fitosociología. 1 herbario

* Plantas leñosas cultivadas. 1 herbario

* Plantas medicinales, aromáticas, insecticidas y alergénicas. 1 herbario

* Plantas medicinales exclusivamente. 1 herbario

* Plantas ornamentales exclusivamente. 1 herbario

* Plantas útiles y plantas dañinas. 1 herbario

* Plantas venenosas exclusivamente. 1 herbario

* Semillas en general. 1 herbario

* Semillas de maíz. 1 herbario

* Violetas exclusivamente. 1 herbario

De todos estos herbarios especializados destacan sobre los demás, a nuestro modo de ver y por su rareza a curiosidad, los herbarios que manejan exclusivamente hongos micorrízicos, esporas y polen, arroz, semillas de maíz y muy especialmente, por el enfoque que tiene, el de hongos de importancia militar (?).

Hay también incluidos dentro de los herbarios generales o regionales algunos muy peculiares por el tratamiento que les da o por la forma en que trabajan. Existen desde luego los "Herbarios Históricos" que generalmente están constituidos por colecciones de naturalistas famosos, tales como Aldrovandi, Mociño y Sessé, etc. Estos herbarios difícilmente están a la disposición del público y por lo general los ejemplares que no son considerados "Tipo" son de escasa utilidad práctica, porque, generalmente y de acuerdo con su antigüedad proporcionan poca información adicional. Estos herbarios son muy útiles por los tipos que pueden contener, pero los demás ejemplares se guardan principalmente por motivos más o menos sentimentales y como testimonio del trabajo, en algunos casos colosal, que desarrollaron sus propietarios originales. Las otras instituciones "excéntricas", por llamarlas de algún modo, incluyen herbarios que nunca determinan sus plantas sino que las envían a instituciones famosas a determinar, esto puede hacerse mediante convenios de diversa índole. Hay también herbarios que se forman exclusivamente a base de comprar ejemplares 
ya determinados; otros más que están constituidos exclusivamente por duplícados de otra institución y que funcionan únicamente como reserva en caso de que la matriz pueda ser destruida natural o artificialmente, y aunque podíamos seguir dando un sinnúmero de ejemplos, mencionemos para finalizar el tema, el caso de los herbarios que sin tomar en cuenta la región en que se encuentran, se dedican exclusivamente a colectar, conservar, estudiar y dar a conocer la flora de una región exótica lejana o particularmente atrayente por otras razones.

\section{5.--LOS USUARIOS}

Nuestra compañera María Teresa García decía en 1953 que "un herbario es una colección de plantas, pero que constituye en sí una fuente de información científica y pragmática. El herbario representa una economía de tiempo y esfuerzo, lo mismo para el estudiante, para el práctico o el aficionado, que para el investigador científico"17 y aunque olvidó mencionar "al público en general" estamos fundamentalmente de acuerdo con ella. Los usuarios de los herbarios son, han sido, y seguramente serán los que nuestra compañera menciona; sin embargo, quisiéramos hablar de otro tipo de usuarios que la historia de los herbarios recuerda con cariño, me refiero a los filósofos y a los políticos que no sólo sabían y entendían lo que eran y hacían los herbarios, sino que dentro de otras muchas actividades y buscando bases más firmes a sus. conocimientos se acercaron a la naturaleza, colectaron plantas sistemáticamente y trabajaron activamente en los herbarios. Entre ellos se encontraron, cada uno a su tiempo, Juan Jacobo Rousseau (1712-1778), Kant (1724-1804), Humboldt (1767-1835), Goethe (1749-1832), Ramos Arizpe (1775-1843), Melchor Ocampo (1815-1861), Aurelio Manrique (1891-1967), etc., etc.

Es una lástima que nuestros actuales filósofos se vayan desligando gradual. mente de la ciencia y una trágica realidad el que la mayoría de los políticos (y hablo a nivel mundial) hayan perdido tan sanas costumbres.

17 García Castañeda, María Teresa. La importancia de un herbario en la Escuela Nacional de Ciencias Biológicas; trabajo leído por su autora en el Seminario de Estudios Biológicos el día 12 de junio de 1953. Boletín del Seminario de Estudios Biológicos. 2: 23-32. 1954. 


\section{6.-LA SISTEMATIZACION DE LOS HERBARIOS}

Después de Linneo y su "Sistema Natural" (1753) fueron apareciendo sucesivamente los sistemas de clasificación de Jussieu (1789); Agustín P. DeCandolle (1813); Endlicher (1836); Brongniart (1846); Bentham y Hooker (1862-1883); von Sachs (1882); Eichler (1883); y Engler y Prantl (18871909), dejando cada uno su impronta en la taxonomía. Paralelamente y dada la confusión que cada sistema traía consigo respecto a los sistemas anteriores, las reglas de nomenclatura fueron variando a partir de 1813 con los trabajos de Agustín P. DeCandolle; la reunión de Londres en 1866 en que se pidió a Alfonso DeCandolle que preparara un proyecto de Leyes de la nomenclatura botánica; mismas que se discutieron y aprobaron en el Primer Congreso Botánico Internacional de París en 1867, en el que se marcó a Linneo como punto de partida; el Congreso Botánico Internacional de Viena en 1905 en el que se aceptaban una serie de excepciones en nombre de taxa bautizados antes de la época de Linneo y se imponía el uso de nombres en Latín; la parcial reunión de botánicos efectuada en 1907 en Estados Unidos como protesta contra los acuerdos de Viena. En esta última se rechazaban las excepciones y la obligariedad del Latín, pero se establecía como extraordinariamente necesario el reconocimiento de un ejemplar "Tipo"18 y finalmente el arduo trabajo conciliador realizado por los Congresos subsiguientes que culminó durante el Congreso de Cambridge (1930) con la adopción mundial del Código Internacional de Nomenclatura Botánica que (supongo) todos conocemos.

Todos estos cambios en la taxonomía y en la nomenclatura así como las sucesivas modificaciones hechas al Código Internacional por las siguientes sesiones del Congreso Botánico Internacional ${ }^{19}$ han marcado, como era de espe. rarse, profundas huellas en el establecimiento y funcionamiento de los herbarios y se ha hecho necesario buscar un medio de sistematización que permita localizarlos y saber de una manera expedita a qué se dedican. Corresponde a Alfonso DeCandolle el mérito de haber sido el primero que intentó esta sistematización en el último capítulo de su "Phytographie" (1880) intitulado "Repartición actual de un gran número de herbarios que sirven como pruebas o explicaciones de las descripciones publicadas". ${ }^{20}$

18 Rickett (op. cit.).

19 Lanjouw, J. et al. eds. International code of botanical nomenclature; Adopted by the ninth International Botanical Congress, Montreal, August 1959. Utrecht, International Bureau for Plant Taxonomy and Nomenclature of the International Association for Plant Taxonomy, 1961. 372 p.

20 DeCandolle, Alphonse. Phytographie. 1880 (original no consultado, citado por Lanjouw In: Lanjouw and Stafleu op. cit. p. 7). 


\section{7.-EL "INDEX HERBARIORUM"}

Basado en el trabajo de DeCandolle, en 1930 el $5^{\circ}$ Congreso Botánico Internacional (Cambridge) creó el "Comité para necesidades taxonómicas urgentes" cuyo primer secretario fue el conocido botánico A. S. Hitchcock, quien comenzó a compilar una lista de autores de descripciones de plantas con la indi. cación de los herbarios en que se encontraban depositados los Tipos.

Después de varios incidentes, en 1935, durante el Congreso de Amsterdam, se tomó la decisión de que la compilación del "Index Herbariorum" debía tener precedencia sobre todos los otros proyectos que había formulado el comité; a la muerte de Hitchcock, J. Lanjouw pasó a ocupar el cargo de Secretario, sin embargo, se tropezó con grandes problemas, el menor de los cuales fue la sempiterna falta de recursos económicos que siempre han sufrido este tipo de instituciones y proyectos, mientras que el mayor obstáculo fue, quizás, la interferencia causada por la Segunda Guerra Mundial. Al terminar ésta y ser creada la UNESCO pudo contarse con apoyo económico y respaldo moral mucho más substanciosos, por lo que después de muchas vicisitudes Lanjouw pudo ver ver coronados sus esfuerzos y con la ayuda de F. A. Stafleu, quien comenzó a colaborar con él en 1950, publicó la primera edición del "Index Herbariorum" en $1952 .{ }^{21}$

-El contenido del "Index Herbariorum" (Parte I).

El "Index Herboriorum" consta de tres secciones:

* Una introducción

* Un prefacio correspondiente a cada una de las ediciones

"Una sección intitulada "Los herbarios del mundo"

Esta última sección está dividido a su vez en cuatro partes:

I. Lista de herbarios

II. Abreviaturas clave para los herbarios

III. Arreglo geográfico de los herbarios

IV. Indice de nombres personales

21 Lanjouw, J. ed. Index Herhariorum; A guide to the location and contents of the world's public herbaria. Uthecht, International Bureau for Plant Taxonomy and Nomencla. tura of the International Association for Plant Taxonomy, 1952. 
Aunque la primera edición del "Index Herbariorum" apareció en 1952, el trabajo para compilarlo había comenzado mucho tiempo antes. En noviembre de 1936 se distribuyó en grandes cantidades y a nivel mundial entre todas las instituciones cuya situación o nombre sugiriera la posibilidad de que contaran con un herbario, una tarjeta circular en la que además de exponer el proyecto y el significado del "Index Herbariorum" se incluian dos preguntas:

"1. ¿Tiene su institución un Herbario?

2. ¿Se interesaría su instituto en colaborar en la manufactura de un Index Herbariorum". ${ }^{22}$

Como ocurre en todas las encuestas no todas las instituciones contestaron, pero los resultados de este primer sondeo fueron bastante satisfactorios.

La segunda circular se envió en junio de 1937, pero la inminencia de la guerra impidió una fácil comunicación, por lo que esta segunda circular fue reimpresa y nuevamente enviada en noviembre de 1947.

En esta última comunicación se invitaba a las instituciones a contestar el cuestionario siguiente ${ }^{23}$ (traducido y reacomodado libremente por el autor):

1. Nombre y dirección postal del herbario.

2. Nombre del Director, Curador u otro miembro del equipo que tenga a su cargo el herbario.

3. Región, país, o grupo taxonómico en que el herbario se especializa.

4. Lista de todos los colectores mencionados en los ejemplares del herbario. En qué país o región han sido hechas estas colectas. Si alguna persona ha realizado más de una colección mencionar dichas colecciones en forma separada. Indicar en la forma más precisa posible la región en que las colectas han sido llevadas a cabo. Si la colección no representa un conjunto de originales, sino un conjunto de duplicados que la institución ha recibido por intercambio o compra de otro herbario marcarlo poniendo una " $\mathrm{D}$ " mayúscula y entre comillas dentro de su información. Dar si es posible el número de especímenes de cada colección.

5. Hacer una lista de autores de los que el herbario posea ejemplares Tipo. Si los ejemplares Tipo de un autor son principalmente de un cierto taxon o de determinado país o región señalar esto entre comillas.

22 Lanjouw and Stafleu (op. cit., p. 9).

23 Lanjouw and Stafleu (op. cit., p. 10). 
6. Contestar si el herbario está dispuesto a mantener intercambio o préstamo de ejemplares.

7. Informar si el herbario posee o está ligado a un jardín botánico.

8. Agregar todos los datos complementarios que se quiera.

Como se puede ver el cuestionario no sólo era muy completo sino quizás excesivamente detallado. Por lo tanto no es de extrañar que muchas instituciones que habían contestado la primer circular no respondieron ésta y que la mayoría de las que sí lo hicieron, proporcionaran los datos en forma vaga o simplemente omitieran contestar algunas preguntas, sobre todo, aquellas de las que era difícil informar lo que se solicitaba.

Para poder dar respuesta a los puntos 4 y 5 sería necesario una labor de titanes por el tiempo y esfuerzo que había que invertir para averiguar, en el propio herbario, todos los detalles que se requerían.

Es obvio que los mayores y más famosos herbarios no tomaron en cuenta dichos puntos.

Es lamentable además, que dada la situación política prevaleciente no tengamos más que unos cuantos datos aislados de los herbarios situados en Europa Oriental, Unión Soviética y China."-4

-Las abreviaturas o claves de los herbarios.

Estas abreviaturas o claves de los herbarios, resultan sumamente útiles y cómodas en el trabajo, pues al igual que los nombres científicos resumen en unas cuantas letras todo un cúmulo de información. Desgraciadamente no existe un código para hacerlas y se han ido dando de una manera totalmente arbitraria. Las primeras las compuso el propio Lanjouw, ${ }^{25}$ pero como muchos de los interesados no estuvieron ce acuerdo con ellas, en la actualidad, se pide a los herbarios que se registran por primera vez que ellos mismos sugieran la abreviatura con que desean ser identificados, aunque esto obviamente ha producido más confusión.

\section{-El arreglo geográfico.}

Este se da en la lista de herbarios por orden alfabético de nombres de ciudades y en la lista geográfica por orden alfabético de los nombres en inglés de los diferentes países.

21 Lanjouw and Stafleu (op. cit., p. 11).

25 Lanjouw, J. (1937, 1939 apud Lanjouw and Stafleu op. cit., pp. 10-11). 
Posteriormente a la primera edición del "Index Herbariorum", han aparecido en diferentes años otras cuantas ediciones más y numerosos suplementos a esta obra, la cual, por su naturaleza misma, debe ser revisada y puesta al día constantemente.

\section{8.--UNOS CUANTOS NUMEROS}

La parte estadística de este trabajo es sin duda la más árida, Lanjouw y Stafleu no incluyen en el "Index Herbariorum" un análisis estadístico, pero nosotros hemos querido hacer un resumen numérico para mayor información de las personas que tengan interés en el asunto. Aclarando que dicho resumen está basando principalmente en la cuarta edición de la obra de los autores citados, ${ }^{26}$ así como en otras fuentes más recientes. ${ }^{27}, 28$

\section{A. Herbarios del Mundo}

La primera circular enviada a un enorme número de Instituciones, fue contestada por 1114 herbarios a los cuales se les dio abreviatura o clave oficial

De esos 1114 herbarios, sólo concontestaron la segunda encuesta, por lo menos parcialmente No la contestaron

B. Dependencia de los herbarios

Herbarios que pertenecen a Instituciones Universitarias, Educativas Autónomas, Privadas o Educativas dependientes del Estado

Herbarios que pertenecen al Estado a través de alguna agencia gubernamental sin estar ligados a la enseñanza

26 Lanjouw and Stafleu (op. cit.).

27 Core, Earl L. Herbarium. In Gray Peter ed. The Encyclopedia of the Biological Sciences. New York, Reinhold, c1961. pp. 476-477.

28 Rzedowski, Jerzy. 1973 (comunicación personal). 
Herbarios privados no ligados a la enseñanza

69

No contestaron esta pregunta

C. Fecha de fundación

Contestaron esta pregunta

No contestaron esta pregunta

659

455

1,114

646

468

1,114

$58 \%$

$42 \%$

$100 \%$

E. Canje y préstamo de ejemplares Herbarios que tienen establecido canje y préstamo de ejemplares, abierto a todas las instituciones similares

Herbarios que tienen establecido canje y préstamo de ejemplares sólo a determinadas y muy selectas instituciones

Herbarios que no tienen estableblecido canje y niegan sistemáticamente el préstamo de sus ejemplares

No contestaron esta pregunta
$6.1 \%$
$26.4 \%$
$100 \%$

L

$59 \%$

$4.1 \%$

$100 \%$

\section{RESUMEN ESTADISTICO}

a) En 1959 se registró la existencia mundial de 1114 herbarios, de los cuales disponemos de datos, aunque sean parciales, de 863 .

b) La mayor parte de los herbarios están ligados con instituciones universitarias o educativas a cualquier otro nivel; en orden de importancia continúan los que dependen directamente del estado, sin estar ligados a actividades de 
enseñanza y finalmente, sólo una corta proporción pertenece a herbarios pri. vados que no están conectados con la enseñanza.

c) Por lo que respecta a la fecha de fundación se encuentran los siguientes datos, tomados en fracciones de tiempos equivalentes a medio siglo a partir de 1500.

De 1500 a 1549 se fundaron los jardines botánicos de Pisa (1543), Padua y Florencia (1545). El primero de ellos estableció (?) un herbario creado por Luca Ghini. Este herbario desapareció y no volvió a existir hasta la primera mital del siglo XIX.

De 1550 a 1559 se establecieron cuatro jardines botánicos, el más importante para nuestra historia es el de Bolonia, en donde con las colecciones de Ulises Aldrovandi se fundó en 1567 el herbario más antiguo que persiste hasta nuestros días, las otras tres instituciones son de menor importancia, fueron creadas como jardines botánicos y no tuvieron un herbario propiamente dicho hasta mucho tiempo después.

De 1600 a 1649 se fundaron dos instituciones de las cuales la más importante fue el Jardín Botánico de París, que posteriormente ha dado lugar a uno de los herbarios más ricos del mundo.

De 1650 a 1699 se fundaron solamente 2 herbarios y

De 1700 a 1749 únicamente tres.

Desde entonces, el auge de los herbarios ha ido en continua expansión y así vemos que

De 1750 a 1799 se establecieron 31 herbarios.

De 1800 a 1849 se crearon 64 .

De 1850 a 1899, 191.

De 1900 a 1949, 327 herbarios.

De 1950 a 1973, 42 más (aunque este último dato seguramente no corresponde a la verdad, pues deben ser muchísimos más, que aún no están registrados).

d) Número de ejemplares.

De los datos proporcionados por 646 herbarios se desprenden las cifras siguientes: los números límite han sido escogidos arbitrariamente pues abarcan una variabilidad sumamente grande que va desde 300 ejemplares hasta más de 6 millones en un solo herbario.

Con menos de 1,000 ejemplares se registraron 14 herbarios de los cuales el menor señaló poseer 300 exsiccata

De 1,000 a 5,000 ejemplares 62 herbarios

De 5,000 a 10,009 ejemplares 58 herbarios 


\section{HISTORIA Y ESTADO ACTUAL DE LOS HERBARIOS}

De 10,000 a 50,000 ejemplares 208 herbarios

De 50,000 a 100,000 ejemplares 94 herbarios

De 100,000 a 250,000 ejemplares 104 herbarios

Die 250,000 a 500,000 ejemplares 50 herbarios

De 500,000 a 1,000.000 de ejemplares 23 herbarios

De 1,000.000 a 2,000.000 de ejemplares 19 herbarios

De 2,000.000 a 3,000.000 de ejemplares 8 herbarios

De 3,000.000 a 4,000.000 de ejemplares 2 herbarios

De 4,000.000 a 5,000.000 de ejemplares 2 herbarios

De 5,000.000 a 6,000.000 de ejemplares 1 herbario

Con más de 6,000.000 de ejemplares 1 herbario

Los cuatro herbarios más grandes del mundo, según el "Index Herbariorum", son en orden creciente: el de Ginebra $(G)$, que entre otras contiene las colecciones de los DeCandolle, con un poco menos de 5 millones de ejemplares; el de París (P) con poco más de 5 millones y los de Kew (K) y Leningrado (LE), ambos con más de 6 millones de ejemplares cada uno. Pero es muy factible que las cifras mencionadas en el párrafo anterior sean únicamente el resultado de cálculos imaginarios bien intencionados; pues, como quedó establecido en un trabajo reciente de Brenan y Carter (32), el Herbario de Kew (K), reputado hasta 1970 como el más grande del mundo con 6-7 millones de ejemplares, contiene en realidad 4,188.000 especímenes y 275,000 tipos.

Debemos hacer notar que en general la antigüedad de un herbario no siempre guarda una relación directa con el número de ejemplares que posee así por ejemplo el herbario de la Academia dei Fisiocritici en Siena (SIAC), fundado en 1691, conserva únicamente 400 ejemplares, el de Salzburgo (SZB) creado en 1800 guarda solamente 2,000 exsiccata; etc., etc.

\section{e) Canje y préstamo de ejemplares}

Es notable el hecho de que un alto porcentaje $(516=46.3 \%)$ de las instituciones haya evadido dar respuesta a esta pregunta; el $53.7 \%$ restante se encuentra dividido en la forma siguiente: 510 instituciones $(45.8 \%)$ declaran tener establecido canje y préstamo abierto con instituciones similares, o botánicos en particular en una forma recíproca y prácticamente sin limitaciones; 67 instituciones, equivalentes al $6 \%$ del total, indican tener serias reservas para el canje y sobre todo para el préstamo, pero éste puede ser obtenido en ciertos casos, cuando la institución o el botánico que lo solicita son ampliamente conocidos en el medio científico y si se corren una serie más o menos compli- 
cada de trámites. Por último 21 instituciones, o sea el $1.9 \%$ del total rechaza categóricamente establecer canjes o prestar sus ejemplares absolutamente a toda persona o institución. De aquí se puede sacar en conclusión que el canje, y sobre todo el préstamo para estudio no es tan general como cualquier espíritu abierto podría pensar.

\section{f) Herbarios y Jardines Botánicos}

De los 863 herbarios que contestaron la encuesta, 210 afirmaron estar actualmente ligados a jardines botánicos, lo cual no quiere decir que existen precisamente 210 jardines botánicos en el mundo, pues muchos de ellos son independientes y no están ligados a ningún herbario; por otra parte se presentan casos en que 2 (TH y TI; TRT y TRTC) y hasta 3 herbarios diferentes ( $\mathrm{P}$, PC, PCU) estén ligados a un solo jardín botánico y viceversa, pues, algunos herbarios manejan 2, (AAH, LI, ZVS) y por lo menos en un caso, un herbario es propietario de 4 jardines botánicos (PE). Podemos comprobar así que los" herbarios como hijos fieles, siguen trabajando con los jardines botánicos que a fin de cuentas y como ya lo hemos visto son las instituciones madres.

g) La distribución de los herbarios

En general, podemos decir que la mayor concentración de herbarios se encuentra en los países más desarrollados. De 151 países reconocidos actualmente $^{29}$ en 94 existen herbarios, Gran Bretaña ocupa el primer lugar con 287 herbarios, corresponde a los Estados Unidos el segundo lugar con 234, Canadá el tercero con 45, la URSS el cuarto con 41 y Francia el quinto con 40 herbarios. Siguiendo esta secuencia, México con 13 herbarios ocupa el onceavo lugar quedando situado entre Australia con 14 y Austria e India, ambos con 12 herbarios cada uno.*

Por lo que respecta a las ciudades en que existen más herbarios, Buenos Aires, Cambridge (Massachusetts), Lisboa y Tolouse comparten los honores del primer lugar con seis herbarios cada una. Finalmente, con relación a la Institución más rica en herbarios, tanto la Harvard University como l'Université de Touluse son poseedoras de cinco herbarios cada una, mismos que se encuentran adscritos a diferentes departamentos de ambas universidades.

29 Cárdenas, Eduardo comp. Diccionario Geográfico 1973; Compendio Mundial. Florida, Editorial América, c1972. pp. 108-116.

* Estas cifras variarán al conocerse el número exacto de herbarios que tienen una función activa en México. 


\section{h) Los enemigos}

Aparte, claro está, de depredación o vandalismo dirigidos personal y concientemente contra la institución, caso que es más bien raro; los principales enemigos de los herbarios son: la humedad, las plagas, el fuego y la guerra.

No encontramos registros de desaparición total y absoluta de herbarios por las dos primeras, humedad y plagas, y pensamos que esto es debido a que siendo los ataques de ellos frecuentes y por lo tanto esperados, los herbarios se mantienen por siempre en pie de lucha, tomando las precauciones y cuidados más adecuados.

Cosa diferentes es el fuego, pues a pesar de todas las recomendaciones que se dan para prevenirlo, ha acabado con un buen número de herbarios. Unicamente tenemos estadísticas de Estados Unidos, en los que por lo menos 7 herbarios fueron totalmente consumidos por el fuego en la primera mitad de este siglo. ${ }^{30},{ }^{31}$

La guerra es cuestión aparte, pues es posiblemente el único enemigo contra el cual los encargados o curadores difícilmente pueden hacer algo.

Cuando comenzamos a elaborar este trabajo, creímos que los registros de los años en que se desarrollaron ambas grandes guerras de este siglo, reflejarían un descenso notable en el número de herbarios fundados durante esos trágicos períodos en comparación con los años de paz, pero un análisis cuidadoso de los datos, realizado comparando entre sí el número de herbarios fundados, año a año, de 1900 a 1950, no mostró ninguna anomalía y la curva que se encontró refleja que el número de instituciones ha sido ascendente, rápido, previsible y $\sin$ caídas durante todo ese medio siglo, y habiendo sido creadas muchas de las instituciones en el seno mismo de los países beligerantes (Alemania, Francia, Italia, etc.), concluimos, aunque nos parece raro, que la guerra en sí no ejerció ninguna influencia sobre la fundación de nuevos herbarios.

Pero, y siempre hay un pero, durante la Segunda Guerra Mundial fueron parcialmente destruidos (en diferentes grados) los importantes herbarios de, por orden alfabético, Berlín (B), Hiroshima (HIRO), Londres (BM), Viena (W) y Würzburg (WB) y absolutamente arrasados los de Bonn (BONN), Leipzig (LZ), Lübeck (LUB) y Marburg (MB) en Alemania; el de Quezon City (PUH) en Filipinas y el de Rovinj (RI) en Yugoslavia.

30 Lawrence, George H. M. Taxonomy of Vascular Plants. New York, Macmillan, 1958. p. 260.

31 Core, Earl L. (op. cit.).

32 Brenan, J. P. M. and R. G. Carter. The counting of the Kew Herbarium. Kew Bull. 26 (3): $423-426.1972$. 
En contra de lo que podía pensarse, la destrucción durante la guerra no partió sólo de los enemigos y de esto da ejemplo el Jardín Botánico de Berlín, totalmente destruido a manos de los propios berlineses, que en su sufrimiento talaron árboles y arbustos tratando de conseguir un poco de combustible para calentarse y acabaron con el resto de las plantas buscando un mínimo de alimento para siquiera engañar al hambre, guisando en algunos casos vegetales exóticos que en otras condiciones a nadie se le hubiera ocurrido que podían ser comestibles, y todo esto se hizo a pesar de los envenamientos ocurridos y de las severas penas a que se condenaba a las personas sorprendidas en esta acción destructiva.

Pero la guerra no tiene sólo en su haber las destrucciones ya mencionadas, en América Latina, el "Herbario del Instituto de La Salle" (BOG) de Bogotá fue borrado del mapa durante la Revolución ocurrida en Colombia en 1948.

En contraste con este triste panorama de destrucción, resulta curioso consignar el dato siguiente, el Herbario de Lae (LAE), en Papua y Nueva Guinea. fue creado durante y gracias a la guerra por un puñado de soldados australianos, enviados a esa región por razones estratégicas y que posiblemente quedaron maravillados ante la hermosura de la flora; no es seguro, pero quizás el cabecilla de los mismos fuera en la vida civil botánico o simplemente aficionado a la botánica.

\section{9.-CONCLUSIONES}

Frecuentemente escuchamos en nuestro medio que los herbarios son instituciones anticuadas, que responden al afán o manía de acumulación de cosas viejas de tal o cual persona, que resultan demasiado onerosos y que, en el mejor de los casos y dado el adelanto actual de la ciencia se ocupan en actividades tan obsoletas como la taxonomía cuyo tiempo ya pasó, debiendo haberse quedado cuando mucho en la segunda mitad del siglo pasado; por lo tanto, se concluye que los herbarios son instituciones muertas. Y lo triste de esta declaración es que no sólo proviene de las autoridades de todo tipo que deberían apoyar el funcionamiento de los herbarios, pero ya hemos visto que la mayoría de los políticos, con muy honrosas excepciones, ha mucho tiempo que perdieron el interés por las cuestiones botánicas habiéndolo enfocado hacia aquellos asuntos más rápida y abundantemente redituables, con poca inversión y en el mínimo de tiempo; sino que, esta opinión también se encuentra en boca de científicos dedicados a otras ramas, mismos que, se supone, deberían apoyar ante autoridades y opinión pública el esfucrzo desarrollado por los herbarios. 
Pero la historia nos enseña todo lo contrario; los herbarios no son instituciones anticuadas por el hecho de no necesitar de complicados instrumentos electrónicos para su trabajo ni porque es imposible o por lo menos innecesario transitorizarlos; son instituciones modernas que, cuando se les da oportunidad, usan y construyen al igual que todos los demás tipos de instituciones, los peldaños que producen el rápido avance de la Ciencia. Es cierto que a veces (y a fin de cuenta somos humanos) se encuentra en ellos la manía de acumulación, pero este defecto lo comparten con las demás instituciones científicas; prueba de ello son los microscopios electrónicos y las computadoras que en algunos casos sólo se adquieren "para estar a la moda" y que luego duermen el sueño de los justos ( ¿o de los injustos?). Por lo que respecta a las cosas viejas que acumula, habrá que reconocer que entre más completa sea una colección, forzosamente sus primeros componentes se irán haciendo viejos; es como en el caso de las bibliotecas, algo de ellas va quedando en la obsolescencia, pero lo bueno, lo verdaderamente bueno, continúa como piedra de toque y finalmente se convierte en clásico; además, haciendo una comparación chusca, hay de cosas viejas a cosas viejas, recordemos al vino.

Que los herbarios resultan demasiado onerosos es una idea que debe haber surgido por el enorme espacio que su instalación requiere, pero es falsa, por lo menos comparando el costo y los resultados obtenidos por un buen herbario, con el costo y los resultados, para dar un solo ejemplo, por una modernísima institución empeñada en la lucha contra el esmog y, finalmente, a los que claman que la taxonomía vegetal es cuestión del siglo pasado, podemos responderles que la taxonomía actual es tan moderna como puede serlo la investigación espacial. Nunca antes en la historia se había llegado al punto que se ha alcanzado, ni nunca, en un período igual de tiempo se habían creado tantos herbarios como los que han aparecido en lo que va de este siglo. Que en México hagamos una taxonomía similar a la que se llevaba a cabo en el siglo pasado en otras partes del mundo, es otra cosa, pues mientras en los países más desarrollados la flora se conoce casi perfectamente, en México llevamos, con respecto a ellos, un atraso de por lo menos 75 años, causado por múltiples razones; pero para poder aprovechar bien nuestros recursos naturales debemos primero conocerlos exactamente y para lograrlo tenemos que recorrer a toda prisa el largo trecho que hemos perdido, haciendo ahora lo que no hicimos hace $75,50,25$ o 10 años y esto deberá suceder siguiendo paso a paso el camino de quienes nos llevan la delantera; utilizando sí, todos los adelantos que la ciencia pone a nuestra disposición, pero sin olvidar que la base firme y los datos con que, por ejemplo, trabajarán las computadọ̣as, sólo podrán obtenerse de un buen herbarịio y de 
un número siempre creciente de colecciones. Pues de nada nos servirá el equipo electrónico o de otro tipo si no tenemos la información fidedigna y nece. saria para que éste trabaje.

Por último deberíamos hablar de los resultados que se esperan de un buen herbario de nuestros tiempos y de las modernas técnicas que allí se aplican, pero esto es ya otra historia y no me corresponde a mí contarla. 